\title{
Effect of DEHP on SCFA Production by Anaerobic Fermentation of Waste Activated Sludge
}

\author{
Rui Gong, ${ }^{1,2}$ Xiang Tang, ${ }^{1,2}$ Changzheng Fan $\mathbb{D}^{1,2}$ Baowei Zhang, ${ }^{1,2}$ and Man Zhou ${ }^{1,2}$ \\ ${ }^{1}$ College of Environmental Science and Engineering, Hunan University, Changsha 410082, China \\ ${ }^{2}$ Key Laboratory of Environmental Biology and Pollution Control (Hunan University), Ministry of Education, \\ Changsha 410082, China \\ Correspondence should be addressed to Changzheng Fan; fancz@hnu.edu.cn
}

Received 14 August 2019; Accepted 4 December 2019; Published 28 February 2020

Academic Editor: Mingzhi Huang

Copyright $\odot 2020$ Rui Gong et al. This is an open access article distributed under the Creative Commons Attribution License, which permits unrestricted use, distribution, and reproduction in any medium, provided the original work is properly cited.

Diethylhexyl phthalate (DEHP) is a common plasticizer in industrial production. Recently, environmental problems caused by microplastics have drawn wide attention. As the microplastics have a large specific surface area, the release rate of the plasticizer from the microplastics to the environment is accelerated. The DEHP in the wastewater enters the wastewater treatment plants (WWTPs) along with the urban pipeline. After DEHP enters the WWTPs, it may affect the anaerobic fermentation with waste activated sludge (WAS) as raw material. So far, there has been no study on the effect of DEHP on anaerobic fermentation of WAS. Our study focused on the impact of exogenous DEHP on WAS anaerobic fermentation, and the results showed that DEHP mainly affects the solubilization stage of sludge anaerobic digestion, but has no significant effect on other stages. It does not affect the total yield and composition of short-chain fatty acids (SCFA). However, DEHP inhibited the solubilization process of WAS anaerobic fermentation, which was mainly manifested by the changes of soluble protein and soluble polysaccharide in the system. The results of the analysis of microbial communities revealed that the addition of DEHP did not change the diversity of microbial communities, but caused a change in the abundance of microbial organisms. DEHP reduced the abundance of acetogen bacteria and increased the abundance of methanogens. This work provides some insights into WAS fermentation systems in the presence of DEHP and helps to gain a better understanding of the potential environmental hazards of microplastics.

\section{Introduction}

Phthalate esters (PAEs) are one of the most widely used groups of industrial products [1-3]. They are primarily used in the production of polyvinyl chloride to make it more gentle and flexible [3-5] and as plasticizers for building materials and furniture, food packaging, and mosquito repellents [5-7]. The exceptional performance of PAEs has promoted them as plasticizers and additives in the plastic production industry for decades [8]. PAEs are not chemically bonded to the polymeric matrix, so they can enter the environment by leaching from final manufacture products or through losses during the manufacturing processes [6], which leads to their ubiquitous occurrence in environmental matrices such as water [5, 9, 10], air [11-13], soil [14-16], sediment [17-19], landfills [20, 21], vegetables [22], and fish [23]. PAEs are attracting more and more attention because they pose a threat to the ecological environment and human health [24].

Some researches [25-27] have reported that industrial products such as microplastics and rubber release large amounts of PAEs, which enter WWTPs through raw wastewater. The preliminary processes in WWTPs can remove some PAEs, and as the most widely used technology in WWTPs, activated sludge process can remove many pollutants, including PAEs. Nevertheless, PAEs are not eliminated after these processes. As the major by-product of sewage treatment, wasted sludge contains a large number of organic pollutants such as PAEs [28]. Lots of PAEs with high molecular weight, like DEHP, are adsorbed into the biological grease and remain in the sludge.

The most commonly found PAEs in sludge are diethylhexyl phthalate (DEHP), benzyl butyl phthalate (BBP), 
dimethyl phthalate (DMP), dibutyl phthalate (DBP), diethyl phthalate (DEP), diisobutyl phthalate (DIBP), and dicyclohexyl phthalate (DCHP) [26], among which DEHP is one of the most widely used plastic additives [29]. It is utilized in a large number of products like consumer products [30], personal care products [31], building materials [32], and so on. Some research found that DEHP is most abundant in sludge $[28,33]$. The concentrations in the sludge of which DEHP was common ranged between 130 and $1094 \mu \mathrm{g} / \mathrm{g}$ dry weight in South Africa's WWTPs [34].

Existing WWTPs are being scaled up to satisfy the needs of a growing city, resulting in more and more WAS [35]. Anaerobic fermentation of WAS can produce energy biogas methane and achieve sludge reduction while minimizing the number of pathogenic microorganisms in the sludge [35-38]. A large number of microorganisms, such as acetogens, hydrogenogens, and methanogens, are linked to the anaerobic fermentation process of WAS $[1,39,40]$. Although anaerobic fermentation is an effective means of dealing with WAS, the existence of a large amount of DEHP in the WAS may affect the microbial community in the WAS anaerobic fermentation system. This may have a negative impact on the process of anaerobic fermentation of sludge to produce SCFA. To the best of our knowledge, the effect of DEHP on the production of SCFA during WAS anaerobic fermentation has not been reported.

Therefore, this study was aimed to illustrate the potential impact of DEHP on the accumulation of SCFA by anaerobic fermentation of WAS. Effect of different DEHP concentrations on SCFA production during WAS anaerobic fermentation was studied. Besides, mechanisms of DEHP impacting SCFA production from WAS were explored. Finally, the long-term effect of DEHP on the microbial communities were investigated. The results of this study will demonstrate the effect of DEHP on the anaerobic fermentation of WAS and have a certain significance on how to treat DEHP in WWTPs.

\section{Materials and Methods}

2.1. Sources of WAS and DEHP. The WAS used in this study was extracted from the secondary sedimentation tank of a municipal WWTPs in Changsha, China. The fresh sludge was shaken evenly and passed through the stainless-steel filter steel mesh $(0.45 \mathrm{~mm})$. The filtered sludge was concentrated at $4^{\circ} \mathrm{C}$ for $24 \mathrm{~h}$ to gain the concentrated sludge used in the study. The relevant properties of concentrated sludge are as follows: $\mathrm{pH} 6.8 \pm 0.1$, total suspended solids (TSS) $17640 \pm 510 \mathrm{mg} / \mathrm{L}$, volatile suspended solids (VSS) $15340 \pm 230 \mathrm{mg} / \mathrm{L}$, total of chemical oxygen demand (COD) $16134 \pm 540 \mathrm{mg} / \mathrm{L}$, soluble chemical oxygen demand (SCOD) $480 \pm 10 \mathrm{mg} / \mathrm{L}$, total carbohydrate $1590 \pm 270 \mathrm{mg} \mathrm{COD} / \mathrm{L}$, and total protein $10340 \pm 460 \mathrm{mg} \mathrm{COD} / \mathrm{L}$. Among them, protein and polysaccharide are two substances with the largest proportion of organic matter in the sludge, and the sum of the two reaches $74 \%$ of the total amount of sludge organic compounds. The fermentation sludge was taken from a long-term reactor of an alkaline pretreatment sludge that had been operating for several months in our lab. The
DEHP used in this study with $95 \%$ purity was obtained from Bidepharm (Shanghai).

2.2. Effects of DEHP at Different Concentrations on the Production of SCFA by Anaerobic Fermentation of WAS. This experiment set up 24 reactors with a working volume of $500 \mathrm{~mL}$. All reactors were divided into two groups and placed in a rocking bed at $35^{\circ} \mathrm{C}$ for 15 days. Various studies have shown that alkali treatment can improve the SCFA production performance of sludge anaerobic fermentation, and alkali treatment as sludge pretreatment has been implemented in many WWTPs [37]. Therefore, both groups of reactors in this experiment were to be established as one that did not control $\mathrm{pH}$ (called Group-A) and one that controlled $\mathrm{pH}$ of 10 (called Group-B), with 12 reactors in each group. Considering the accumulation of DEHP in the environment, the concentration of DEHP will be higher in the future environment, so it is necessary to design DEHP with high concentration for experiments. The concentration of DEHP in the experiment was finally determined to be $20 \mathrm{ppm}, 50 \mathrm{ppm}$, and $100 \mathrm{ppm}$, respectively. At the same time, a reactor without additional DEHP was created and defined as a control group.

$300 \mathrm{~mL}$ concentrated sludge was inoculated to each reactor. An additional dose of DEHP was added to each reactor to make the DEHP level in the 4 reactors as 0 , $20 \mathrm{ppm}, 50 \mathrm{ppm}$, and $100 \mathrm{ppm}$. At the same time, the reactor of Group-B used hydrochloric acid or sodium hydroxide to adjust the $\mathrm{pH}$ to 10 . Then, all reactors were filled with nitrogen for $5 \mathrm{~min}$, which was quickly sealed with a rubber plug. After this, all reactors were placed in a shaking table with a rotation speed of $120 \mathrm{rpm}$ and a temperature of $35^{\circ} \mathrm{C}$ for $15 \mathrm{~d}$. The SCFA concentration in each reactor was measured daily.

\subsection{Accessing the Impact of DEHP on the Sludge Solubilization} Process. In this set of experiments, 12 reactors were set up. $300 \mathrm{~mL}$ of concentrated sludge was added to each reactor. One of the reactors did not add any additional chemicals as a control group. For the experimental group, different doses of DEHP were added to the remaining three reactors to achieve a DEHP concentration of $20 \mathrm{ppm}, 50 \mathrm{ppm}$, and $100 \mathrm{ppm}$, respectively. The influence of DEHP on the sludge solubilization process will be investigated by analyzing the concentration changes of soluble COD (SCOD), soluble polysaccharide, and protein in the fermentation broth of four reactors and the morphological changes of extracellular polymer substances (EPS).

2.4. To Evaluate the Effects of DEHP on Hydrolysis, Acidogenesis, Acetogenesis, and Methanogenesis Processes. WAS anaerobic fermentation mainly goes through the following processes: solubilization, hydrolysis, acidogenesis, acetogenesis, and methanogenesis. In order to further explore the influence of DEHP on WAS anaerobic fermentation, a series of batch experiments will be set up to assess the influence of DEHP on each process. In this series of experiments, there 
were 48 serum bottles with a working volume of $500 \mathrm{ml}$. These serum bottles were divided into four groups with four in each. Experiments were carried out by adding common standard substances to reaction systems. These four experimental groups were named as Test-A, Test-B, Test-C, and Test-D.

Test-A: in Test-A bovine serum albumin (BSA) and glucan were used as model substrates to evaluate the effect of DEHP on the hydrolysis process. In this set of experiments, $30 \mathrm{~mL}$ of fermented sludge was received in each serum bottle and $260 \mathrm{~mL}$ of tap water was added. In addition, $1.8 \mathrm{~g}$ of BSA and $0.47 \mathrm{~g}$ of glucan were added into each serum bottle of the experimental group as model substrates. One of these reactors did not add additional chemicals to serve as a control reactor, and to the remaining three reactors were added different doses of DEHP. The DEHP levels in the remaining reactors were $20 \mathrm{ppm}, 50 \mathrm{ppm}$, and $100 \mathrm{ppm}$, respectively. The fermentation conditions of the sludge in these reactors were indistinguishable from the abovementioned experiments.

Test-B: Test-B was used to evaluate the effect of DEHP on the acidogenesis process. In this set of experiments, except for the difference of the model substrate, the relevant operation process is not different from Test-A. The difference between the model substances was that the amino acid replaces the BSA in Test-A, and the dextran in Test-A was replaced by glucose. The added amount of amino acid was $1.8 \mathrm{~g}$, and the added amount of glucose was $0.47 \mathrm{~g}$.

Test-C: this test was used to evaluate the effect of DEHP on the acetogenesis process. The operating conditions of this experimental group are not different from those of Test-A. Only sodium butyrate was utilized to replace BSA and glucan. The addition amount of sodium butyrate was $1.8 \mathrm{~g}$.

Test-D: Test-D was used to assess the effect of DEHP on the methanogenesis process. There were no differences in reaction conditions between Test-D and Test-C except for the model substance added in the reactors. Sodium acetate replaced sodium butyrate in Test-C, and $1.8 \mathrm{~g}$ of sodium acetate was added in the reactors.

2.5. Long-Term Semicontinuous Reactors for Measurement of Microbial Community. In this experiment, two long-term semicontinuous reactors were established. The reactors contain $300 \mathrm{~mL}$ of alkaline pretreated sludge ( $\mathrm{pH}$ adjusted to 10), and additional DEHP was added to achieve a concentration of $100 \mathrm{ppm}$ in the reactor. In addition to the operations described below, the semicontinuous reactors have no difference in operating conditions from the previously set experiments. According to the SCFA production experiment mentioned above, when the DEHP concentration in the sludge was $100 \mathrm{ppm}$ in the Group-B, the maximum SCFA yield measured was 5 days after the reaction. Therefore, the residence time of the sludge in Group-B was artificially controlled for 5 days. $60 \mathrm{ml}$ of sludge fermentation broth was manually extracted from the reactors every day. An equal amount of alkaline pretreated sludge was then added to the reactors. All reactors continued to operate for two months. Two months later, the microbial community structure and microbial abundance in the two reactors' sludge systems were analyzed.

2.6. Analysis Methods. The DEHP was determined by GCMS. All glassware used in the whole determination process should be washed and rinsed with distilled water, soaked in acetone for 1 hour, and then dried and cooled to room temperature for later use. Firstly, $20 \mathrm{~mL}$ samples were drawn from the set reactor with a syringe and added to the centrifugal tube. After centrifugation at a speed of $5000 \mathrm{rpm}$ for $5 \mathrm{~min}$, the supernatant was discarded. Then centrifuged samples were dried in a vacuum drying cabinet for $48 \mathrm{~h}$. After that, $0.5 \mathrm{~g}$ (accurate to $0.1 \mathrm{mg}$ ) of the sample was weighed and transferred to a $50 \mathrm{~mL}$ Erlenmeyer flask. First, $1 \mathrm{~mL} n$-hexane was added into the Erlenmeyer flask, and the mixture was swirled for 2 min by using a vortex instrument. $5 \mathrm{~mL}$ acetonitrile was added in the well-mixed solution and swirled for $1 \mathrm{~min}$. After this, the evenly mixed solution in the conical flask was placed in the ultrasonic instrument to extract for $20 \mathrm{~min}$. Then, the supernatant was obtained by centrifugation at $5000 \mathrm{rpm}$ for $5 \mathrm{~min} .5 \mathrm{~mL}$ acetonitrile was added to the supernatant, and the previous extraction step was repeated to merge the supernatant. Finally, it was evaporated under $1 \mathrm{~mL}$ by weak nitrogen at $40^{\circ} \mathrm{C}$, and then $6 \mathrm{~mL}$ acetonitrile was added, which was mixed in a vortex. The mixture was purified with an activated SPE column. The activated SPE column was achieved by adding $5 \mathrm{~mL}$ dichloromethane and $5 \mathrm{~mL}$ acetonitrile to the column and discarding the effluent. After the SPE column was activated, the obtained mixture was supplemented by the SPE column, and the outflow was collected. $5 \mathrm{~mL}$ acetonitrile was added to collect the outflow and combine the two collected outflows, and $1 \mathrm{ml}$ acetone was added. It was blowed to nearly dry at $40^{\circ} \mathrm{C}$, and then $\mathrm{n}$-hexane was utilized in constant volume to $2 \mathrm{~mL}$ followed by vortex mixing for GC-MS analysis.

The relevant parameters of GC-MS are set as follows: injection port temperature: $260^{\circ} \mathrm{C}$. Temperature program: the initial column temperature was $60^{\circ} \mathrm{C}$ for $1 \mathrm{~min}$; the temperature was raised to $220^{\circ} \mathrm{C}$ for $1 \mathrm{~min}$ at a rate of $20^{\circ} \mathrm{C} /$ $\mathrm{min}$; then the temperature was raised to $250^{\circ} \mathrm{C}$ for $1 \mathrm{~min}$ at a rate of $5^{\circ} \mathrm{C} / \mathrm{min}$; and finally, the temperature was raised to $290^{\circ} \mathrm{C}$ at a rate of $20^{\circ} \mathrm{C} / \mathrm{min}$ for $7.5 \mathrm{~min}$. Carrier gas: high purity helium gas (>99.999\% purity), flow rate: $1.0 \mathrm{~mL} / \mathrm{min}$. Injection mode: no shunt injection. Injection: $1 \mu \mathrm{L}$. Ionization mode: electron bombardment ionization source (EI); ionization energy: $70 \mathrm{eV}$; transmission line temperature: $280^{\circ} \mathrm{C}$; ion source temperature: $230^{\circ} \mathrm{C}$; and monitoring mode: selection scanning (SIM). Solvent delay: $7 \mathrm{~min}$.

Standard methods were used for the determination of TS, VS, TSS, VSS, COD, and SCOD [41, 42]. The glucosebased phenol-sulfuric method and BSA-based Lowry-Folin method are used to determine soluble polysaccharides and soluble proteins in the fermentation broth [43]. And SCFA is primarily used in gas chromatography to determine the methods mentioned in references [44]. The analytical method for free amino acids is the ninhydrin colorimetry [45]. 
Different EPS fractions were extracted by thermal extraction [46]. Briefly, $45 \mathrm{~mL}$ of the sludge sample is taken from the reactor. The sample was centrifuged at $4500 \mathrm{~g}$ for $10 \mathrm{~min}$, and the resulting supernatant was S-EPS. The sludge particles in the tube were diluted with $\mathrm{NaCl}(0.05 \%)$ solution (preheated to $70^{\circ} \mathrm{C}$ ) to an initial volume of $45 \mathrm{~mL}$, which was then vortexed on a vortex mixer for $1 \mathrm{~min}$ and finally centrifuged at $4500 \mathrm{~g}$. The supernatant obtained after centrifugation was thought to be a loosely bound EPS (LB-EPS). The remaining sludge precipitated in the tube was diluted with $\mathrm{NaCl}(0.05 \%)$ solution to the original volume of $45 \mathrm{~mL}$, and it was put into a water bath at $60^{\circ} \mathrm{C}$ for $30 \mathrm{~min}$ and finally centrifuged at $4500 \mathrm{~g}$ for $10 \mathrm{~min}$. The supernatant collected after centrifugation was reckoned to be a tightly bound EPS (TB-EPS).

2.7. Statistical Analyses. All the experiments in this study were in triplicate, and the results were expressed as mean \pm standard deviation.

\section{Results and Discussion}

3.1. Effect of DEHP on SCFA Production by Sludge Anaerobic Fermentation. Figure 1(a) shows the effect of different levels of DEHP on the accumulation of SCFA by anaerobic fermentation of WAS. The total production of SCFA with different DEHP levels displayed similar trends. From the 1st day to the 5th day, the SCFA content continues to rise and reached the maximum on the 5th day (control: $4098 \mathrm{mg}$ $\mathrm{COD} / \mathrm{g}$ VSS). Then, the production of SCFA gradually decreased until day 8 , and the total SCFA content in all reaction systems increased slightly and reached the second peak on day 9. Then, the production of SCFA continued to decrease in the remaining time. The two increasing trends of SCFA production during anaerobic fermentation are inconsistent with the results of previous studies [47, 48]. Further data analysis showed that the concentration of DEHP in the reaction system did not significantly change the yield of SCFA.

Alkali treatment is an ordinary sludge pretreatment method. It can enhance sludge anaerobic fermentation to produce SCFA, which has been widely utilized in WWTPs. It is needful to further investigate the effect of DEHP on the production of SCFA by anaerobic fermentation of alkaline pretreated sludge. The change of SCFA production during anaerobic fermentation of alkaline pretreated sludge with dissimilar levels of DEHP is shown in Figure 1(b). Observing Figures 1(a) and 1(b), we found that the variation of SCFA production in the two reaction systems was similar. The SCFA production in Group-B reached two peaks on the 5th day (control: $10066 \mathrm{mg} \mathrm{COD} / \mathrm{g}$ VSS) and 9th day (100 ppm: $6958 \mathrm{mg} \mathrm{COD} / \mathrm{g}$ VSS), respectively. Compared with GroupA, SCFA generated from Group-B was more stable and less volatile. Moreover, the addition of DEHP did not cause regular changes in SCFA yield in Group-A and Group-B.

To better comprehend how DEHP affects sludge anaerobic fermentation to produce SCFA, the components of SCFA including acetic acid, propionic acid, iso-butyric acid, $n$-butyric acid, iso-valeric acid, and $n$-valeric acid were further calculated. Figures $1(\mathrm{c})$ and $1(\mathrm{~d})$ showed the percentage of each component of total SCFA on the 5th day. The results have shown that DEHP neither affects the production of SCFA from WAS anaerobic fermentation nor does it alter the components. Regardless of the dose of DEHP, it has no impact on the components of SCFA production in the system. In Group-A, the main components of SCFA were acetic acid and propionic acid, accounting for $28 \%$ and $33 \%$, respectively. There was a major change in the proportion of SCFA in Group-B compared with Group-A. Compared with Group-A, the main components of SCFA in Group-B were acetic acid and propionic acid. Compared with Group-A, the acetic acid increased rapidly from $28 \%$ to $47 \%$ and propionic acid decreased from $33 \%$ to $19 \%$ in Group-B. The alkaline pretreated sludge will increase the solubilization of organic matter in the sludge, thereby greatly promoting the total amount of SCFA produced by the anaerobic fermentation of the sludge. Compared with Group-A, the amount of SCFA produced in Group-B increased significantly, but the addition of DEHP did not cause changes between Group-B. This fully demonstrates that the increase in the total amount of SCFA produced in Group-B is due to the effect of alkali pretreatment, which makes full use of the organic matter solubilization by alkali pretreatment and is not affected by DEHP. This indicated that the addition of DEHP would cause slight fluctuations in the sludge system, but it had no significant effect on the SCFA production and the components of SCFA generated by WAS anaerobic fermentation.

As well, WAS anaerobic fermentation would go through several steps: solubilization, hydrolysis, acidogenesis, acetogenesis, and methanogenesis. Therefore, it is necessary to further investigate the effects of DEHP on other processes of sludge anaerobic fermentation.

\subsection{DEHP Inhibited the Sludge Solubilization Process.} Solubilization is the first process of WAS anaerobic fermentation. In this process, macromolecular substances in sludge fermentation broth will dissolve, so soluble COD is deemed to be an indicator to evaluate the level of organic matter in the sludge fermentation broth. The changing trend of soluble COD in the reaction system caused by different levels of DEHP in 5 days is shown in Figure 2(a). The soluble COD showed an increasing trend first and then decreasing with the reaction time. The experimental group (the reaction system containing different levels of DEHP) produced less soluble COD than the control group (the reaction system without DEHP), indicating that DEHP has a certain inhibiting effect on the solubilization process of WAS, but the degree of inhibition is not related to the concentration level of DEHP. Proteins and polysaccharides are important components of COD. Therefore, in order to further explore how DEHP inhibits the solubilization of sludge, we also investigated the changes of soluble proteins and soluble polysaccharides, as illustrated in Figures 2(b) and 2(c), respectively. The content of soluble proteins and soluble 


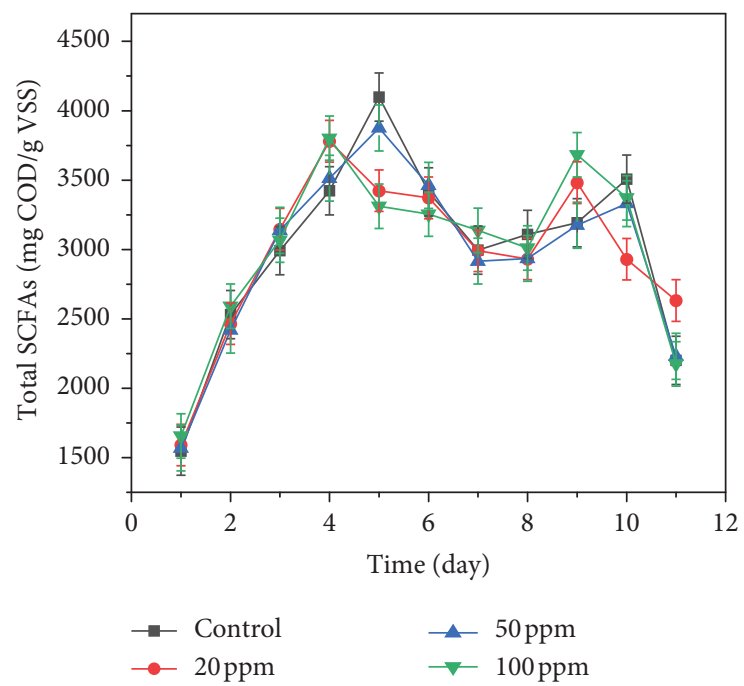

(a)

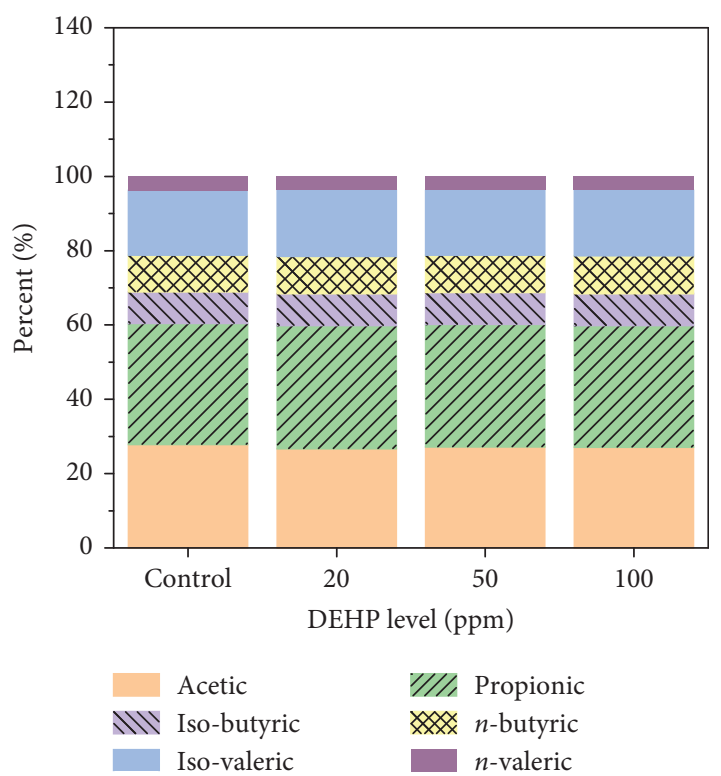

(c)

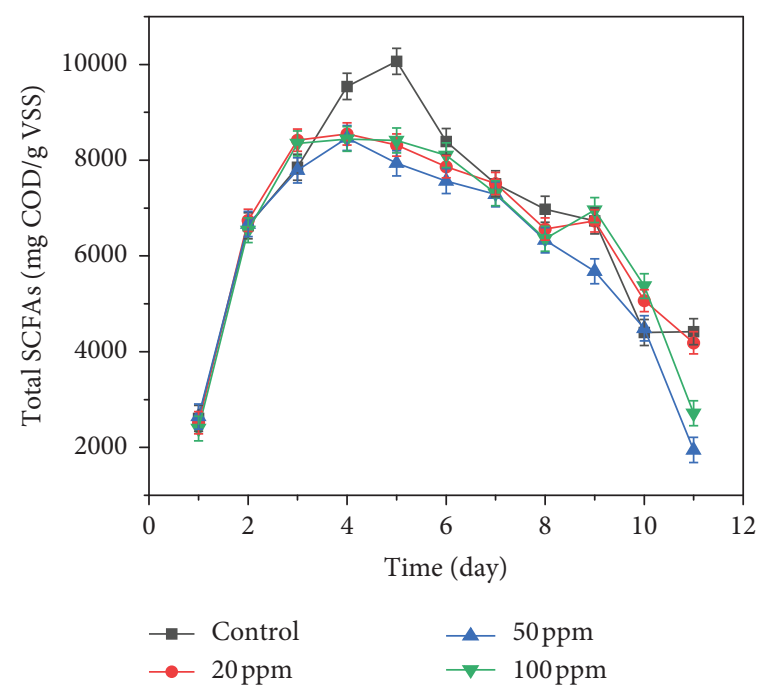

(b)

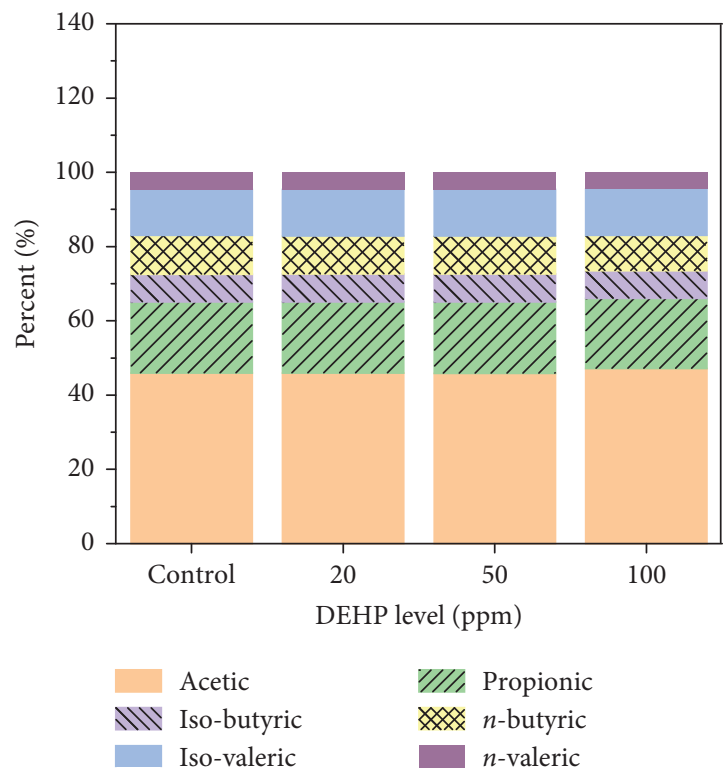

(d)

FIGURE 1: The effect of DEHP on the total SCFA yield and the ratio of each SCFA under optimal conditions of anaerobic fermentation sludge. (a) SCFA yield without alkali pretreatment; (b) SCFA yield with alkaline pretreatment; and (c) the ratio of each SCFA on 5 days without alkali pretreatment; (d) The ratio of each SCFA on 5 days with alkaline pretreatment. Error bars represent standard deviations of triplicate tests.

polysaccharides in the experimental group was basically lower than those in the control group. From the 1st day to the 3th day, the content of soluble protein showed an increase first and then a decrease. On the 4th day, a second increase in protein content occurred in the reactors. The phenomenon of two peaks was similar to the effect of DEHP on SCFA production described in Section 3.1. Also, soluble polysaccharides were basically consistent with the trend of soluble proteins, which showed an increase first and then a decrease. On the 2 nd day of the reaction, the soluble polysaccharide content in the control group $(122.87 \mathrm{mg} / \mathrm{g}$ VSS) was significantly higher than that in the experiment group $(59.50 \mathrm{mg} / \mathrm{g}$ VSS). Changes in soluble COD were mainly attributed to changes in soluble protein and soluble polysaccharide. The effect of DEHP on the solubilization stage of sludge anaerobic digestion showed a slight inhibition in general. It was known from the experiment that the effect of DEHP concentration on sludge solubilization does not show certain regularity.

3.3. Effect of DEHP on Extracellular Polymers of Sludge. The effects of DEHP on three different forms of extracellular polymers of sludge, namely S-EPS, LB-EPS, and TB-EPS were discussed. The contents of polysaccharides and proteins in the three extracellular polymers were detected, as 


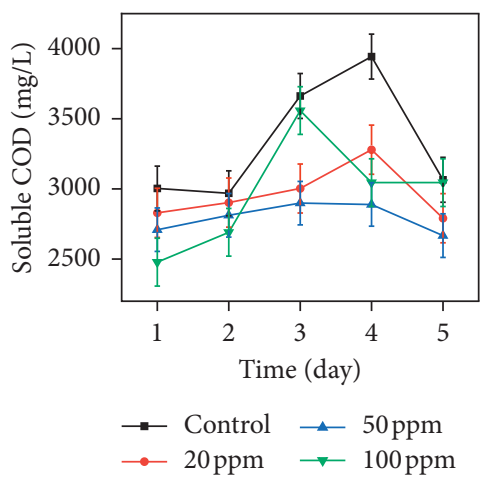

(a)

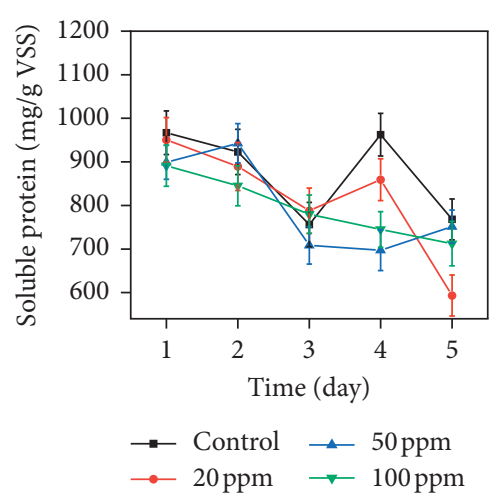

(b)

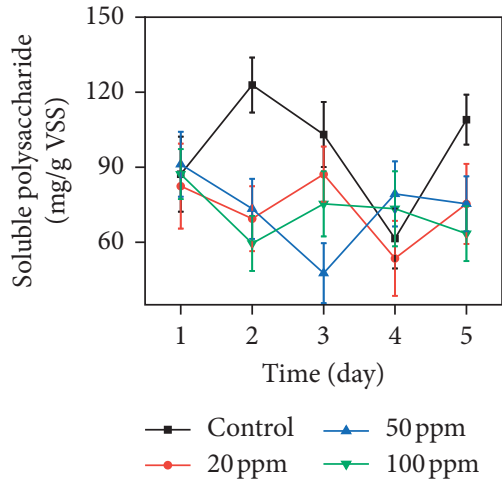

(c)

FIgURE 2: Soluble substances' concentration in 5 days of fermentation time measured in the fermentation reactors with alkaline pretreatment in the presence of different DEHP contents. (a) Soluble COD; (b) soluble proteins; and (c) soluble polysaccharide. Error bars represent standard deviations of triplicate tests.

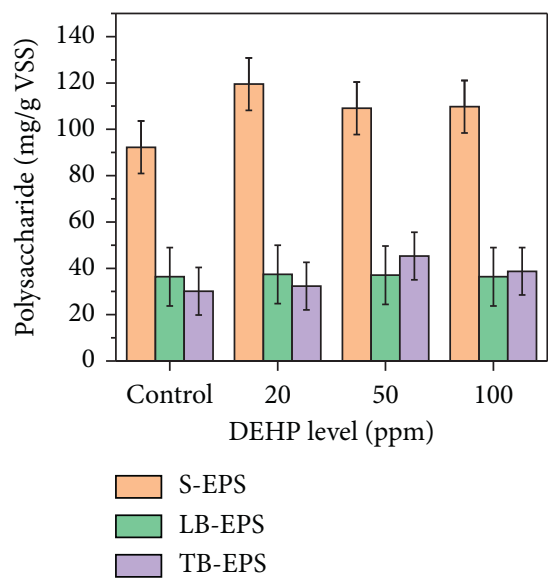

(a)

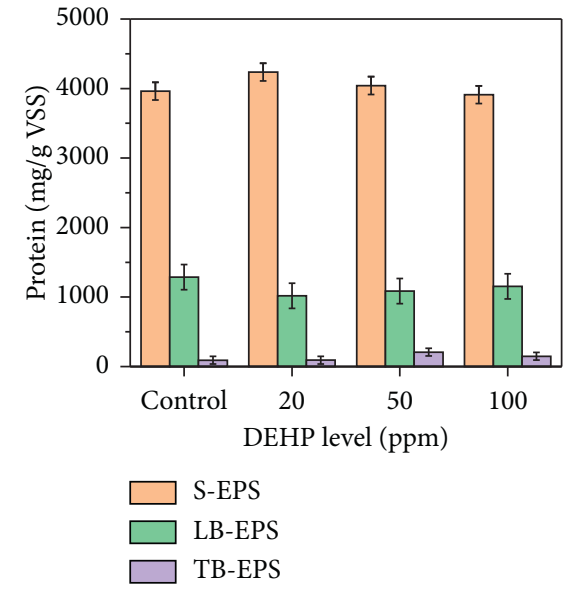

(b)

Figure 3: Effect of DEHP content on sludge EPS at the fermentation time of $3 \mathrm{~d}$. (a) Polysaccharide and (b) protein. Error bars represent standard deviations of triplicate tests.

shown in Figures 3(a) and 3(b), respectively. It indicates that polysaccharides and proteins in different shapes of EPS have similar change trends. The polysaccharide and protein in S-EPS showed a decreasing trend after a small increase, especially the increase of polysaccharide from $92 \mathrm{mg} / \mathrm{g}$ VSS to $119 \mathrm{mg} / \mathrm{g}$ VSS. The maximum value appears in the reaction system with a DEHP concentration of $20 \mathrm{ppm}$. The trend of polysaccharides and proteins in LB-EPS was similar to S-EPS. But, its increase and decrease ranges are small, which basically can be regarded as without an apparent change. Polysaccharide levels are stable at around $37 \mathrm{mg} / \mathrm{g}$ VSS, while protein levels vary between $1100 \mathrm{mg} / \mathrm{g}$ VSS and $1300 \mathrm{mg} / \mathrm{g}$ VSS. The polysaccharides and proteins in TB-EPS showed a similar change trend with S-EPS. When the DEHP level was $50 \mathrm{ppm}$ in the system, the maximum value of polysaccharides and proteins appeared. The highest content was $45 \mathrm{mg} / \mathrm{g}$ VSS for polysaccharide and $207 \mathrm{mg} / \mathrm{g}$ VSS for protein.
Furthermore, the sludge extracellular polymer was subjected to three-dimensional fluorescence scanning. The resulting EEM spectrum is shown in Figure 4. EEM fluorescence spectroscopy is commonly used to determine structural changes in extracellular polymers and fermentation broth [44]. By observation, the measured extracellular polymer EEM mainly includes two peaks, peak A and peak B. These two peaks represent tyrosine-like and tryptophanlike proteins, respectively $[49,50]$.

Figures 4(a)-4(d) represent the EEM spectrum of S-EPS. As can be observed in Figure 4, S-EPS contains only peak A, the fluorescence intensity of Figures 4(a)-4(d) is compared, and the difference between them is very small. This showed that the addition of DEHP has little effect on S-EPS, and there was no obvious regularity. The three-dimensional fluorescence analysis showed that both the LB-EPS and TBEPS sludge extracellular polymers contained two peaks, peak 


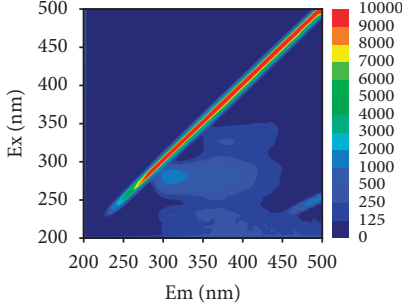

(a)

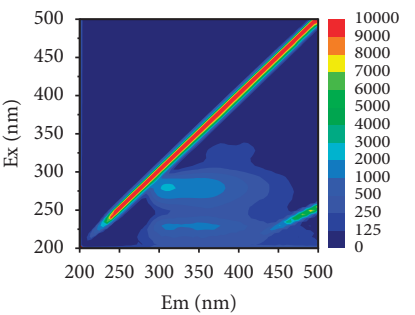

(e)

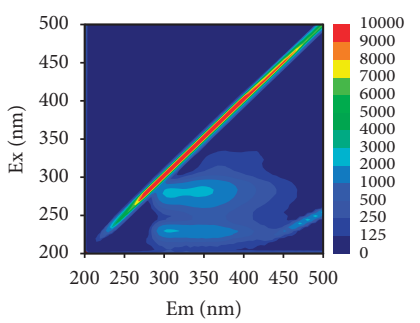

(i)

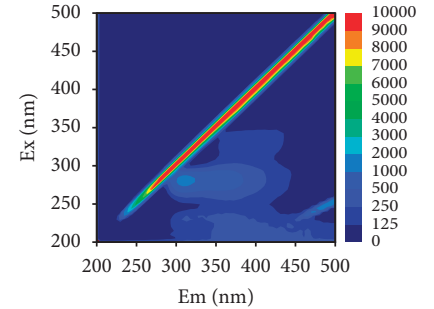

(b)

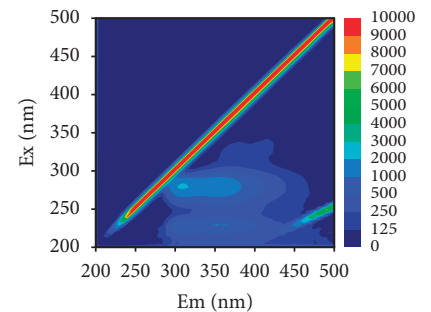

(f)

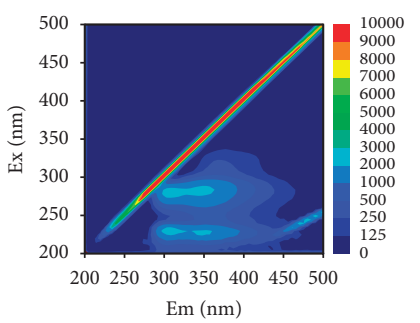

(j)

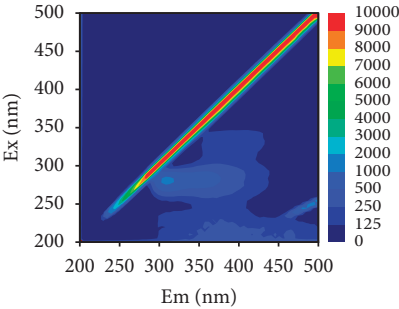

(c)

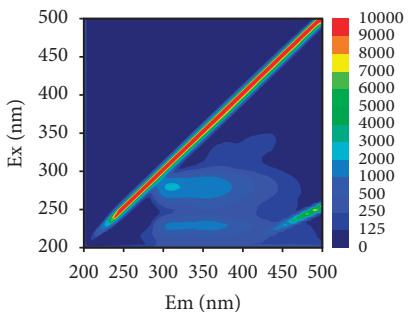

(g)

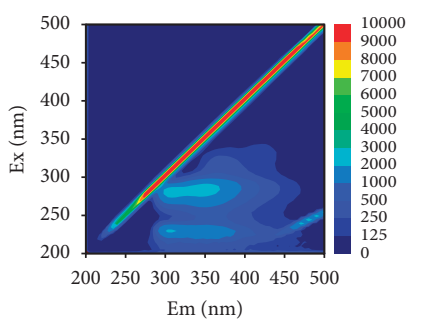

(k)

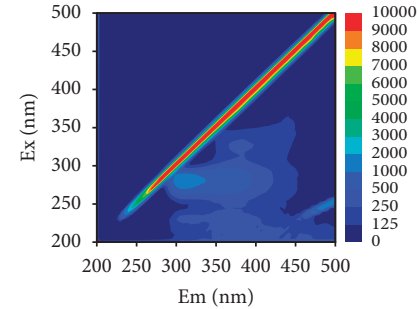

(d)

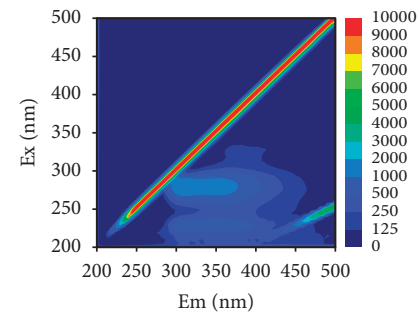

(h)

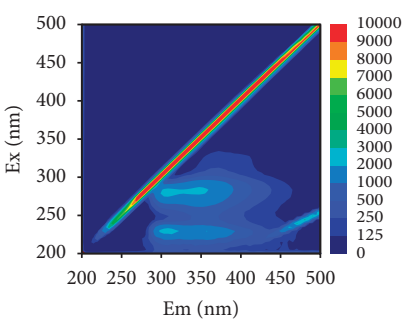

(l)

FIGURE 4: EEM spectra of extracellular polymers. (a) S-EPS: control; (b) S-EPS: DEHP: 20 ppm; (c) S-EPS: DEHP: 50 ppm; (d) S-EPS: DEHP: 100 ppm; (e) LB-EPS: control; (f) LB-EPS: DEHP: 20 ppm; (g) LB-EPS: DEHP: 50 ppm; (h) LB-EPS: DEHP: 100 ppm; (i) TB-EPS: control; (j) TB-EPS: DEHP: 20 ppm; (k) TB-EPS: DEHP: 50 ppm; and (l) TB-EPS: DEHP: 100 ppm.

A and peak B. Also, the EEM spectra of LB-EPS and TB-EPS showed similar trends. More obviously, when the DEHP concentration in the system was $100 \mathrm{ppm}$, the fluorescence intensity measured by LB-EPS and TB-EPS was significantly lower than that of the control group (without DEHP). When the concentration of DEHP was $20 \mathrm{ppm}$ and $50 \mathrm{ppm}$, the fluorescence intensity of LB-EPS and TB-EPS was different from that of the control group, that is, slightly higher than the control group. Especially at a DEHP concentration of $50 \mathrm{ppm}$, the fluorescence intensity of peak $A$ and peak $B$ in LB-EPS and TB-EPS was the largest. The trend changes obtained here are consistent with the previously described experimental results for DEHP affecting sludge extracellular polymers.

3.4. The Effect of DEHP on Hydrolysis, Acidogenesis, Acetogenesis, and Methanogenesis Processes. Hydrolysis refers to the hydrolysis of organic matter into small molecular substances. For example, glucan is converted to glucose and proteins are converted to amino acids [48]. According to the content of hydrolyzed protein and polysaccharide in the reaction system, the degree of material hydrolysis in the reaction system was judged. As can be seen from Figure 5(a), the experimental group showed that DEHP slightly promoted the hydrolysis process. Compared with the control group, the content of the hydrolyzed protein has an increasing tendency. However, the hydrolysis process of anaerobic fermentation is not affected by the dose of DEHP.

Figure 5(b) shows the effect of DEHP on the acidogenesis process. In this work, the trend of amino acid content was measured. The total amount of amino acids showed an increasing trend first and then decreasing within 3 days. At the same time, the concentration of DEHP did not cause changes and fluctuation in the total amount of amino acids in the reaction system. The total amount of amino acids produced in the experimental group was basically the same as that of the control group. This phenomenon indicates that the exogenous substance DEHP does not affect the acidogenesis process of WAS anaerobic fermentation.

After sludge anaerobic fermentation has undergone solubilization and hydrolysis processes, it will involve multiple biological processes, all of which are related to the accumulation of acetic acid. The effect of DEHP on the acetogenesis process of sludge anaerobic fermentation was further explored. The content of butyrate produced in the first 3 days of the reaction system was measured. It can be seen from Figure 5(c) that the butyric acid content shows a rising trend first and then decreasing. Its maximum value was $8651 \mathrm{mg} / \mathrm{L}$ at DEHP concentration of $20 \mathrm{ppm}$. However, from the overall trend, the addition of DEHP does not affect the acetogenic process of WAS anaerobic fermentation. 


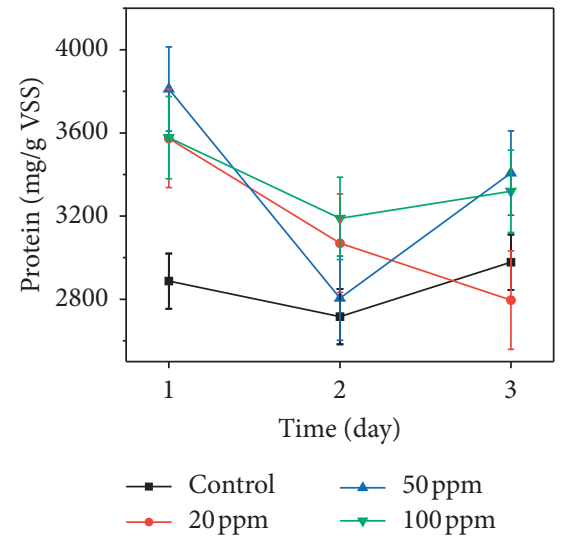

(a)

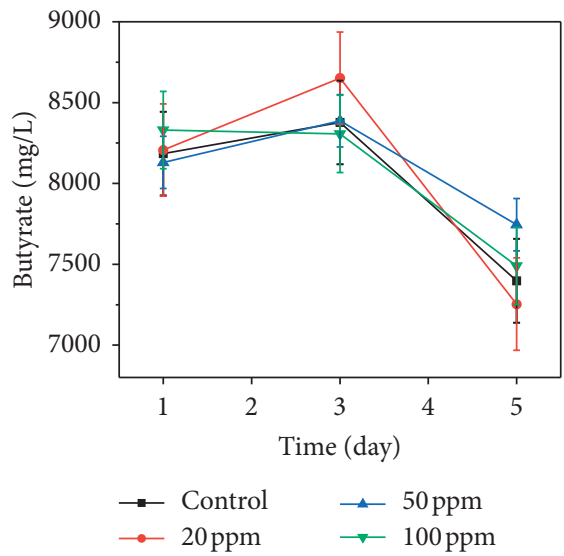

(c)

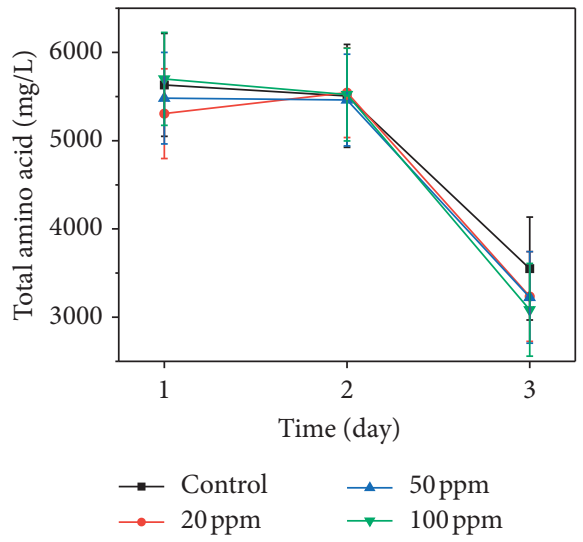

(b)

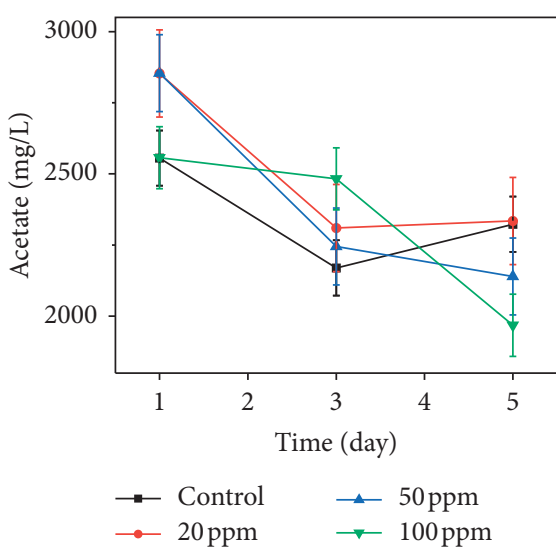

(d)

Figure 5: Concentration of various indicator substances in water distribution experiment concentration in $5 \mathrm{~d}$ of fermentation time measured in the fermentation reactors with alkaline pretreatment in the presence of different DEHP contents. (a) Protein; (b) total amino acid; (c) butyrate; and (d) acetate. Error bars represent standard deviations of triplicate tests.

Methanogenesis is one of the important processes of sludge anaerobic fermentation, and the methane produced can be collected and used as a clean energy source. As shown in Figure 5(d), the acetic acid production of the experimental group was higher than that of the control group at the initial stage of the reaction. However, as the reaction progressed, the acetic acid content of the experimental group decreased sharply and was lower than that of the control group. This indicates that the presence of DEHP in the system slows down the WAS anaerobic fermentation methanogenesis process as the reaction proceeds. It is speculated that the possible reason is that the methanogenic archaea are relatively sensitive. When DEHP enters the sludge system as a toxic exogenous substance, it causes certain damage to the methanogen, thus affecting the WAS anaerobic fermentation of the methanogenesis process.

In summary, the effect of DEHP on sludge anaerobic fermentation is not obvious, but there is a certain interference effect. Therefore, it is necessary to further explore whether the presence of DEHP will affect the microbial community structure and abundance in the sludge system.
3.5. Effects of DEHP on Microbial Community. The performance of WAS anaerobic fermentation is closely related to the microbial community structure and microbial abundance of the sludge system $[35,51]$. In order to better explore whether DEHP has a potential influence on microbial community structure, the Illumina Hiseq16S DNA genes technique was used to conduct a comparative analysis of microbial communities in two long-term reactors (control reactor: without DEHP; DEHP reactor: DEHP concentration is $100 \mathrm{ppm})$. The number of operational taxonomic units (OTUs) detected in the control reactor and DEHP reactor was approximately the same (123 vs. 121 ), which indicated that DEHP had no significant effect on the structure and diversity of microbial communities.

The structure of microorganisms and the distribution of bacterial populations at the phylum level are shown in Figure 6. Firmicutes, Bacteroidetes, Cloacimonetes, Proteobacteria, and Actinobacteria were the dominant bacteria in the two reactors at the phylum level. In anaerobic conditions, many microorganisms in the Firmicutes, Proteobacteria, and Actinobacteria can degrade organic 


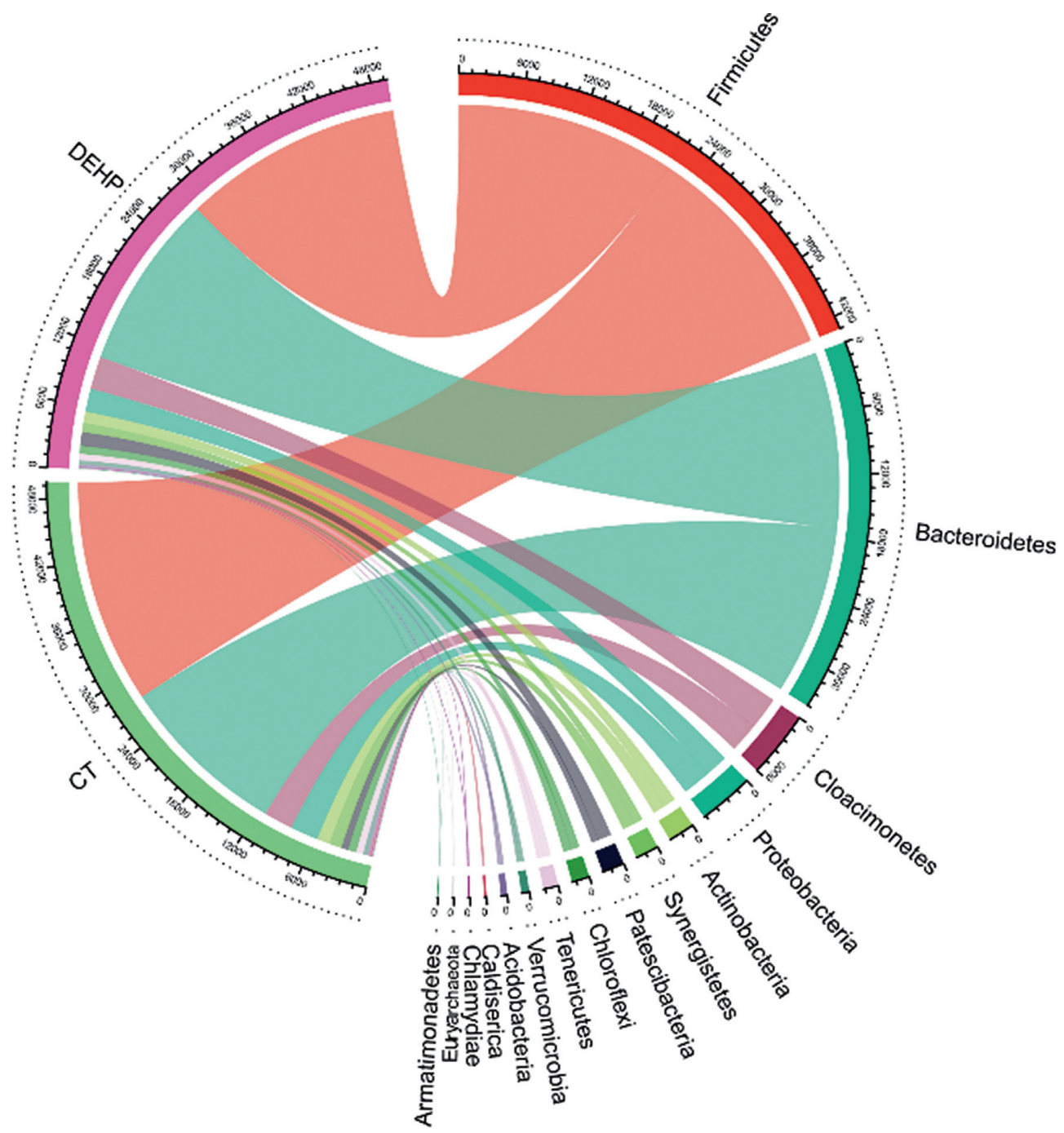

FIGURE 6: The structure of microorganisms and the distribution of bacterial population at the phylum level.

compounds and produce SCFA. Firmicutes and Proteobacteria, with acetic acid as the main products, are reported to be the main producers of SCFA $[44,51]$. With the addition of DEHP, the microbial abundance of Firmicutes, Proteobacteria, and Actinobacteria was decreased, which indicated that the presence of a certain concentration of DEHP in the sludge reaction system could affect the degradation of organic matter in the sludge and inhibit the production of SCFAs. The decline in the abundance of related microbial associated with degradation of pollutants was consistent with the conclusions of previous experiments. DEHP has inhibited the solubilization process of the fermentation broth, which may be since the content of organic compounds in the fermentation broth was affected by the decrease in the proportion of related microbial communities.

Figure 7 showed the distribution of bacterial populations at the genus-level in both long-term reactors. As shown in Figure 7, the microbial abundance in the two reactors were significantly different at the genus-level. It was found that the microbial community structure of the two reactors has changed significantly by comparing the microbial community structure. The proportion of some microorganisms in the two reactors has changed considerably. The abundance of TOP30 bacteria varies slightly in the two reactor systems. Some of these bacteria have changed significantly in proportion. The relative abundance of Blvii28_wastewater sludge_group sp. decreased from $8.02 \%$ (control reactor) to $4.95 \%$ (DEHP reactor). The abundance of Sedimentibacter and Fastidiosipila associated with SCFA production showed a downward trend. Sedimentibacter decreased from 3.64\% (control reactor) to $1.8 \%$ (DEHP reactor), and Fastidiosipila decreased from $3.24 \%$ (control reactor) to $1.17 \%$ (DEHP reactor). It is worth noting that Syntrophomonas, which presented in both reactors, can rapidly degrade accumulated volatile acids and produce methane [52]. Its relative abundance of control reactor and DEHP reactor were $3.47 \%$ and $3.91 \%$, respectively. This gave a reasonable explanation for the methane production of the experimental group in the initial stage of DEHP, which was higher than that of the control group. 


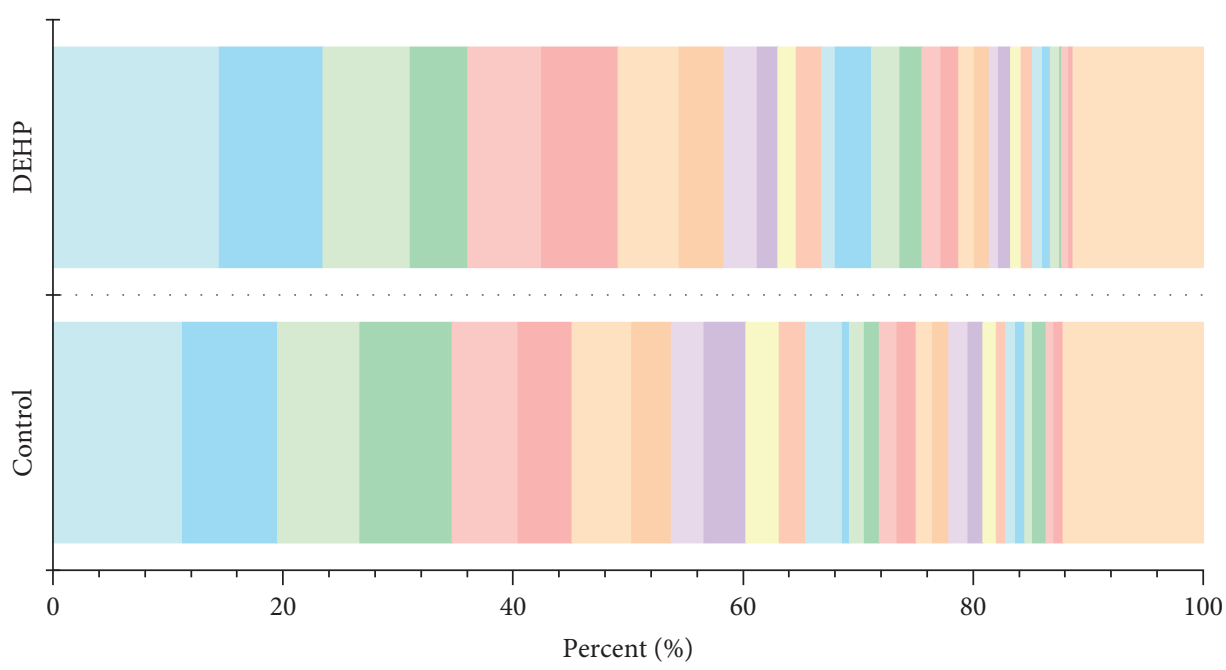

Others
Lentimicrobium
f_uncultured_bacterium_Unclassified
f_Ambiguous_taxa_Unclassified
f_Pedosphaeraceae_Unclassified
Methylocystis
f_Clostridiaceae_1_Unclassified
f_Ruminococcaceae_Unclassified
Erysipelothrix
Pseudomonas
f_Synergistaceae_Unclassified
Acholeplasma
f_Anaerolineaceae_Unclassified
Lamia
Proteiniborus
[Eubacterium]_coprostanoligenes_group

\begin{tabular}{|l}
\hline Petrimonas \\
f_Family_XIV_Unclassified \\
Fastidiosipila \\
Lutispora \\
f_Syntrophomonadaceae_Unclassified \\
Sedimentibacter \\
Ruminococcaceae_UCG-010 \\
Syntrophomonas \\
Christensenellaceae_R-7_group \\
Unclassified_Unclassified \\
LNR_A2-18 \\
Blvii28_wastewater-sludge_group \\
Proteiniphilum \\
Tissierella \\
f_Rikenellaceae_Unclassified
\end{tabular}

FIGURE 7: The structure of microorganisms and the distribution of bacterial population at the genus level.

\section{Conclusion}

When the DEHP level in the sludge system was $20 \mathrm{ppm}$, $50 \mathrm{ppm}$, and $100 \mathrm{ppm}$, the presence of DEHP did not affect the production of SCFA from WAS anaerobic fermentation, regardless of the yield or composition of SCFA. Further real sludge experiment had shown that DEHP has no significant effect on the hydrolysis, acidogenesis, acetogenesis, and methanogenesis processes of anaerobic fermentation of WAS. But, the presence of DEHP inhibited the process of solubilization. According to the results of microbial community analysis, the presence of DEHP in the sludge system may be caused by the reduced abundance of Firmicutes and Proteobacteria. During the observation of the experimental data, it was found that when DEHP involved in the anaerobic fermentation process of WAS, the experimental data measured by the system of DEHP had some fluctuation, which was not well explained in this study. However, the phenomenon of reaction system disorder caused by the addition of DEHP deserves further study and discussion. Therefore, the next research work will be carried out around this phenomenon, so that we can better comprehend the impact of DEHP on WAS anaerobic fermentation.

\section{Data Availability}

The data used to support the findings of this study have not been made available because this research has not yet been fully completed, and we will next investigate the fluctuation of the anaerobic fermentation of the waste activated sludge caused by DEHP.

\section{Conflicts of Interest}

The authors declare that there are no conflicts of interest regarding the publication of this paper.

\section{Authors' Contributions}

Rui Gong and Xiang Tang contributed equally to this paper.

\section{Acknowledgments}

This work was supported by the Key Laboratory of Environmental Biology and Pollution Control, Hunan University, and financially supported by the National Natural Science Foundation of China (51679084). 


\section{References}

[1] X. Gao, J. Li, X. Wang et al., "Exposure and ecological risk of phthalate esters in the Taihu Lake basin, China," Ecotoxicology and Environmental Safety, vol. 171, pp. 564-570, 2019.

[2] S. Jobling, T. Reynolds, R. White, M. G. Parker, and J. P. Sumpter, "A variety of environmentally persistent chemicals, including some phthalate plasticizers, are weakly estrogenic," Environmental Health Perspectives, vol. 103, no. 6, pp. 582-587, 1995.

[3] Y.-M. Lee, J.-E. Lee, W. Choe et al., "Distribution of phthalate esters in air, water, sediments, and fish in the Asan Lake of Korea," Environment International, vol. 126, pp. 635-643, 2019.

[4] J. L. Lyche, A. C. Gutleb, A. Bergman et al., "Reproductive and developmental toxicity of phthalates," Journal of Toxicology and Environmental Health, Part B, vol. 12, no. 4, pp. 225-249, 2009.

[5] J. Wang, Y. Luo, Y. Teng, W. Ma, P. Christie, and Z. Li, "Soil contamination by phthalate esters in Chinese intensive vegetable production systems with different modes of use of plastic film," Environmental Pollution, vol. 180, pp. 265-273, 2013.

[6] H. Fromme, T. Küchler, T. Otto, K. Pilz, J. Müller, and A. Wenzel, "Occurrence of phthalates and bisphenol A and F in the environment," Water Research, vol. 36, no. 6, pp. 1429-1438, 2002.

[7] C. A. Staples, D. R. Peterson, T. F. Parkerton, and W. J. Adams, "The environmental fate of phthalate esters: a literature review," Chemosphere, vol. 35, no. 4, pp. 667-749, 1997.

[8] Y. He, Q. Wang, W. He, and F. Xu, "The occurrence, composition and partitioning of phthalate esters (PAEs) in the water-suspended particulate matter (SPM) system of Lake Chaohu, China," Science of the Total Environment, vol. 661, pp. 285-293, 2019.

[9] K. K. Selvaraj, G. Sundaramoorthy, P. K. Ravichandran, G. K. Girijan, S. Sampath, and B. R. Ramaswamy, "Phthalate esters in water and sediments of the Kaveri River, India: environmental levels and ecotoxicological evaluations," Environmental Geochemistry and Health, vol. 37, no. 1, pp. 8396, 2015.

[10] N. Domínguez-Morueco, S. González-Alonso, and Y. Valcárcel, "Phthalate occurrence in rivers and tap water from central Spain," Science of the Total Environment, vol. 500-501, pp. 139-146, 2014.

[11] M. J. Teil, M. Blanchard, and M. Chevreuil, "Atmospheric fate of phthalate esters in an urban area (Paris-France)," Science of the Total Environment, vol. 354, no. 2-3, pp. 212-223, 2006.

[12] W. J. G. M. Peijnenburg and J. Struijs, "Occurrence of phthalate esters in the environment of The Netherlands," Ecotoxicology and Environmental Safety, vol. 63, no. 2, pp. 204-215, 2006.

[13] S. Kong, Y. Ji, L. Liu et al., "Spatial and temporal variation of phthalic acid esters (PAEs) in atmospheric PM10 and PM2.5 and the influence of ambient temperature in Tianjin, China," Atmospheric Environment, vol. 74, pp. 199-208, 2013.

[14] S. Kong, Y. Ji, L. Liu et al., "Diversities of phthalate esters in suburban agricultural soils and wasteland soil appeared with urbanization in China," Environmental Pollution, vol. 170, pp. 161-168, 2012.

[15] P. Plaza-Bolaños, J. A. Padilla-Sánchez, A. Garrido-Frenich, R. Romero-González, and J. L. Martínez-Vidal, "Evaluation of soil contamination in intensive agricultural areas by pesticides and organic pollutants: south-eastern Spain as a case study," Journal of Environmental Monitoring, vol. 14, no. 4, pp. 1181-1188, 2012.

[16] H. Wang, H. Liang, and D.-W. Gao, "Occurrence and risk assessment of phthalate esters (PAEs) in agricultural soils of the Sanjiang Plain, northeast China," Environmental Science and Pollution Research, vol. 24, no. 24, pp. 19723-19732, 2017.

[17] L. Kang, Q.-M. Wang, Q.-S. He et al., "Current status and historical variations of phthalate ester (PAE) contamination in the sediments from a large Chinese lake (Lake Chaohu)," Environmental Science and Pollution Research, vol. 23, no. 11, pp. 10393-10405, 2016.

[18] C. Chen, C. Chen, and C. Dong, "Distribution of phthalate esters in sediments of Kaohsiung Harbor, Taiwan soil and sediment contamination," Chia-Nan Annual Bulletin, vol. 37, pp. 88-95, 2011.

[19] W. Liu, J. Chen, J. Hu, X. Ling, and S. Tao, "Multi-residues of organic pollutants in surface sediments from littoral areas of the Yellow Sea, China," Marine Pollution Bulletin, vol. 56, no. 6, pp. 1091-1103, 2008.

[20] R. He, B.-H. Tian, Q.-Q. Zhang, and H.-T. Zhang, "Effect of Fenton oxidation on biodegradability, biotoxicity and dissolved organic matter distribution of concentrated landfill leachate derived from a membrane process," Waste Management, vol. 38, pp. 232-239, 2015.

[21] Y. Kalmykova, N. Moona, A.-M. Strömvall, and K. Björklund, "Sorption and degradation of petroleum hydrocarbons, polycyclic aromatic hydrocarbons, alkylphenols, bisphenol A and phthalates in landfill leachate using sand, activated carbon and peat filters," Water Research, vol. 56, pp. 246-257, 2014.

[22] J. Wang, G. Chen, P. Christie, M. Zhang, Y. Luo, and Y. Teng, "Occurrence and risk assessment of phthalate esters (PAEs) in vegetables and soils of suburban plastic film greenhouses," Science of the Total Environment, vol. 523, pp. 129-137, 2015.

[23] L. Xiao, X. Nie, and D. Pan, "Analysis of PAEs in muscle tissue of freshwater fish from fishponds in Pearl River Delta," Journal of Environment and Health, vol. 3, 2008.

[24] T. Deng, X. Xie, J. Duan, and M. Chen, "Di-(2-ethylhexyl) phthalate induced an increase in blood pressure via activation of ACE and inhibition of the bradykinin-NO pathway," Environmental Pollution, vol. 247, pp. 927-934, 2019.

[25] C. Berardi, D. Fibbi, E. Coppini et al., "Removal efficiency and mass balance of polycyclic aromatic hydrocarbons, phthalates, ethoxylated alkylphenols and alkylphenols in a mixed textiledomestic wastewater treatment plant," Science of the Total Environment, vol. 674, pp. 36-48, 2019.

[26] Q. Zhu, J. Jia, K. Zhang, H. Zhang, and C. Liao, "Spatial distribution and mass loading of phthalate esters in wastewater treatment plants in China: an assessment of human exposure," Science of the Total Environment, vol. 656, pp. 862-869, 2019.

[27] R. G. Sawers, "o-Phthalate derived from plastics' plasticizers and a bacterium's solution to its anaerobic degradation," Molecular Microbiology, vol. 108, no. 6, pp. 595-600, 2018.

[28] Y. Wang, Y. Li, Z. H. Wen, and L. Z. Liu, "Occurrence of phthalate esters in sewage sludge from wastewater treatment plants in Shanghai, China," in Environmental Engineering, Pts 1-4, H. Li, Q. Xu, and H. Ge, Eds., pp. 926-929, Trans Tech Publications Ltd., Durnten-Zurich, Switzerland, 2014.

[29] C. Molino, S. Filippi, G. A. Stoppiello, R. Meschini, and D. Angeletti, "In vitro evaluation of cytotoxic and genotoxic effects of di(2-ethylhexyl)-phthalate (DEHP) on European sea bass (Dicentrarchus labrax) embryonic cell line," Toxicology in Vitro, vol. 56, pp. 118-125, 2019. 
[30] D. Ran, Y. Luo, Z. Gan, J. Liu, and J. Yang, "Neural mechanisms underlying the deficit of learning and memory by exposure to di(2-ethylhexyl) phthalate in rats," Ecotoxicology and Environmental Safety, vol. 174, pp. 58-65, 2019.

[31] D. Koniecki, R. Wang, R. P. Moody, and J. Zhu, "Phthalates in cosmetic and personal care products: concentrations and possible dermal exposure," Environmental Research, vol. 111, no. 3, pp. 329-336, 2011.

[32] M. Wittassek, H. M. Koch, J. Angerer, and T. Brüning, "Assessing exposure to phthalates - the human biomonitoring approach," Molecular Nutrition \& Food Research, vol. 55, no. 1, pp. 7-31, 2011.

[33] D. İ. Çifci, C. Kınacı, and O. A. Arikan, "Occurrence of phthalates in sewage sludge from three wastewater treatment plants in Istanbul, Turkey," CLEAN - Soil, Air, Water, vol. 41, no. 9, pp. 851-855, 2013.

[34] T. Salaudeen, O. Okoh, F. Agunbiade, and A. Okoh, "Fate and impact of phthalates in activated sludge treated municipal wastewater on the water bodies in the Eastern Cape, South Africa," Chemosphere, vol. 203, pp. 336-344, 2018.

[35] W. Wei, Q.-S. Huang, J. Sun, J.-Y. Wang, S.-L. Wu, and B.-J. Ni, "Polyvinyl chloride microplastics affect methane production from the anaerobic digestion of waste activated sludge through leaching toxic bisphenol-A," Environmental Science \& Technology, vol. 53, no. 5, pp. 2509-2517, 2019.

[36] D. Wang, Y. Wang, Y. Liu et al., "Is denitrifying anaerobic methane oxidation-centered technologies a solution for the sustainable operation of wastewater treatment plants?," Bioresource Technology, vol. 234, pp. 456-465, 2017.

[37] Y. Wang, D. Wang, Y. Liu et al., “Triclocarban enhances shortchain fatty acids production from anaerobic fermentation of waste activated sludge," Water Research, vol. 127, pp. 150-161, 2017.

[38] Y. Wang, D. Wang, Q. Yang, G. Zeng, and X. Li, "Wastewater opportunities for denitrifying anaerobic methane oxidation," Trends in Biotechnology, vol. 35, no. 9, pp. 799-802, 2017.

[39] K. Liu, Y. Chen, N. Xiao, X. Zheng, and M. Li, "Effect of humic acids with different characteristics on fermentative shortchain fatty acids production from waste activated sludge," Environmental Science \& Technology, vol. 49, no. 8, pp. 4929-4936, 2015.

[40] L. Feng, Y. Chen, and X. Zheng, "Enhancement of waste activated sludge protein conversion and volatile fatty acids accumulation during waste activated sludge anaerobic fermentation by carbohydrate substrate addition: the effect of pH," Environmental Science \& Technology, vol. 43, no. 12, pp. 4373-4380, 2009.

[41] J. Liu, Q. Yang, D. Wang et al., "Enhanced dewaterability of waste activated sludge by $\mathrm{Fe}(\mathrm{II})$-activated peroxymonosulfate oxidation," Bioresource Technology, vol. 206, pp. 134-140, 2016.

[42] A. P. H. Association, A. W. W. Association, W. P. C. Federation, and W. E. Federation, Standard Methods for the Examination of Water and Wastewater, American Public Health Association, Washington, DC, USA, 1915.

[43] O. H. Lowry, N. J. Rosebrough, A. L. Farr, and R. J. Randall, "Protein measurement with the Folin phenol reagent," The Journal of Biological Chemistry, vol. 193, no. 1, pp. 265-275, 1951.

[44] Q. Xu, X. Li, R. Ding et al., "Understanding and mitigating the toxicity of cadmium to the anaerobic fermentation of waste activated sludge," Water Research, vol. 124, pp. 269-279, 2017.

[45] H. U. Bergmeyer, "IFCC methods for the measurement of catalytic concentrations of enzymes," Clinica Chimica Acta, vol. 105, no. 1, pp. 147-154, 1980.
[46] X. Y. Li and S. F. Yang, "Influence of loosely bound extracellular polymeric substances (EPS) on the flocculation, sedimentation and dewaterability of activated sludge," Water Research, vol. 41, no. 5, pp. 1022-1030, 2007.

[47] J. Luo, Q. Zhang, L. Wu et al., "Improving anaerobic fermentation of waste activated sludge using iron activated persulfate treatment," Bioresource Technology, vol. 268, pp. 68-76, 2018.

[48] X. Liu, Q. Xu, D. Wang et al., "Unveiling the mechanisms of how cationic polyacrylamide affects short-chain fatty acids accumulation during long-term anaerobic fermentation of waste activated sludge," Water Research, vol. 155, pp. 142-151, 2019.

[49] B. Frølund, R. Palmgren, K. Keiding, and P. H. Nielsen, "Extraction of extracellular polymers from activated sludge using a cation exchange resin," Water Research, vol. 30, no. 8, pp. 1749-1758, 1996.

[50] A. Ramesh, D. J. Lee, and J. Y. Lai, "Membrane biofouling by extracellular polymeric substances or soluble microbial products from membrane bioreactor sludge," Applied Microbiology and Biotechnology, vol. 74, no. 3, pp. 699-707, 2007.

[51] W. Wei, Q. Huang, J. Sun, X. Dai, and B.-J. Ni, "Revealing the mechanisms of polyethylene microplastics affecting anaerobic digestion of waste activated sludge," Environmental Science \& Technology, vol. 53, no. 16, pp. 9604-9613, 2019.

[52] M. Kim, M. Abdulazeez, B. M. Haroun, G. Nakhla, and M. Keleman, "Microbial communities in co-digestion of food wastes and wastewater biosolids," Bioresource Technology, vol. 289, Article ID 121580, 2019. 